\title{
A Prospective Study of Minimally Invasive Anterolateral Approach in the Supine Position Versus Direct Anterior Approach Total Hip Arthroplasty. Early results
}

\author{
Hongwen Liu \\ Panzhihua Central Hospital \\ Li Yin \\ Panzhihua Central Hospital \\ Jiao Li \\ Panzhihua Central Hospital \\ Shaojiang Liu \\ Panzhihua Central Hospital \\ Qifeng Tao \\ Panzhihua Central Hospital \\ Jie Xu ( $\nabla$ jiexu2913@163.com ) \\ Fujian Provincial Hospital
}

\section{Research Article}

Keywords: Total Hip Arthroplasty, Minimally Anterolateral Approach, Direct Anterior Approach, Early results

Posted Date: February 28th, 2022

DOI: https://doi.org/10.21203/rs.3.rs-1377451/v1

License: (c) (1) This work is licensed under a Creative Commons Attribution 4.0 International License. Read Full License 


\section{Abstract \\ Background}

Both of Minimally Anterolateral Approach (MIS-ALA) and Direct Anterior Approach (DAA) have been reported to be advantageous in Total Hip Arthroplasty (THA), reducing postoperative pain and permit quicker rehabilitation by preserving muscle insertions. There is little literature concern the difference of them. Therefore, we conducted a prospective study to assess the difference of early clinical outcomes, radiological, and patient-reported outcomes between the two minimally invasive approaches of MIS-ALA and DAA.

\section{Methods}

The subjects were 98 patients, with 50 in the MIS-ALA group and 48 in the DAA group. Patients with complete data were evaluated preoperatively and postoperatively at 2, 6 and 12 weeks. Clinical measurements including the ability to climb stairs and walk, a 6 minutes walk test (6MWT) distances, the Forgotten Joint Scale (FJS-12), Japanese Orthopaedic Association (JOA) Hip scores, radiological evaluation, and complications data were collected.

\section{Results}

There were no significant differences in clinical outcomes and implant alignments between MIS-ALA and DAA groups. In regards to patient-reported outcomes, the FJS-12 was significantly higher in the MIS-ALA group than in the group DAA at 2 and 6 weeks postoperatively. However, there was no significant difference in the FJS-12 between the two groups at 12 weeks after surgery. The differences also included shorter operative times, less blood loss, lower $\mathrm{Hb}$ drop, and fewer blood transfusions in the MIS-ALA group. The incidence of lateral femoral cutaneous nerve (LFCN) neuropraxia after surgery was 7 (14.6\%) in the DAA group, zero occurred in the MIS-ALA group. One fracture was identified with each group and managed conservatively

\section{Conclusion}

Both MIS-ALA and DAA approaches yielded excellent and similar early clinical outcomes. However, better patient-reported outcomes could be achieved by MIS-ALA THA. The MIS-ALA was a safer approach for shorter operative times, less blood loss, lower Hb drop, fewer blood transfusions and LFCN neuropraxia compared with DAA. A longer follow-up is needed to examine differences between these procedures.

\section{Introduction}

Primary Total Hip Arthroplasty (THA) is generally considered to be one of the most successful surgeries in modern medicine ${ }^{1,2}$. Which leads to pain relief, functional recovery, and improvement in the quality of life. Technological advancements matched with low revision rates have seen candidates undergoing THA becoming younger ${ }^{3}$. The demands of patients for THA are not only related to the resolution of pain and restoration of function but also a faster recovery ${ }^{4}$.

Total hip arthroplasty (THA) performed through a minimally invasive approach provides earlier pain relief and functional recovery, and has recently been widely used ${ }^{5-7}$. The direct anterior approach (DAA) causes less soft tissue trauma and shorter hospitalization than conventional approaches owing to it follows intermuscular interval, separates the anatomic interval between the tensor fasciae latae and the sartorius muscles to reach the hip joint ${ }^{8,9}$. On the other hand, the minimally anterolateral approach (MIS-ALA) separates the intermuscular plane between the tensor fasciae latae and the gluteus medius muscles, which was the modified Watson-Jones approach and was first reported by Bertin et al ${ }^{10}$ in 2004 . Both of MIS-ALA and DAA have been reported to be advantageous in THA, reducing postoperative pain and permit quicker rehabilitation by preserving muscle insertions ${ }^{11-13}$.

However, to our best knowledge, there have been limited comparative studies available presenting clinical outcomes performed by a single surgeon via the MIS-ALA and DAA approaches. To devote to a better understanding of the effects of MIS-THA on clinical and other parameters, we conducted a prospective study to assess the difference of early clinical outcomes, radiological, and patient-reported outcomes between the two minimally invasive approaches of MIS-ALA and DAA.

\section{Materials And Methods}

\section{Patients}

From January 2017 to January 2018, we recruited 98 patients (98 hips) with hip osteoarthritis of terminal stage who underwent primary unilateral cementless THA. The inclusion criteria for the study were patients had an underlying diagnosis of hip osteoarthritis, age between 20 and 80 years, American Society of Anaesthesiologists grade (ASA) less than or equal to 3, and a body mass index (BMI) not more than $30 \mathrm{~kg} / \mathrm{m}^{2}$. The exclusion criteria were who had a previous history of the hip surgery, rheumatoid arthritis, neuromuscular disease, deformity in limb joints other than the hip joint. Patients were randomized to MIS-ALA ( $n=50)$ or DAA groups $(n=48)$, using computer-generated cards.

\section{Operative Techniques}


All surgeries were performed by one senior surgeon, who started MIS-ALA and DAA in 2011, and the surgeon had practiced more than 100 THAs via MISALA and more than 200 THAs via DAA before this study.

In group MIS-ALA, the patient was placed supine on the fracture table, an oblique skin incision about 9-11 cm was made depend on the build of the patient, extending distally from $2 \mathrm{~cm}$ lateral and distal to the anterior superior iliac spine and ending at the top of the greater trochanter. The subcutaneous tissue and fascia were opened. The intermuscular interval between the anterior border of gluteus medius and the tensor fasciae latae was entered by blunt dissection with the insertion of the index finger until the femoral neck was reached. The vessels crossing this interval was ligated, tensor fascia latae, glutei medius, and minimus was not be split or detached. The capsule was divided in a circular arc fashion along the base of the femoral neck and preserved. The acetabulum was prepared and the acetabular component was inserted using a traditional fashion. For preparation of the femur, the surgeon places the involved lower limb in external rotation, adduction, and the fracture table was extended for $15^{\circ}$ to provide a hyperextension position. An elevating retractor was inserted posterior to the greater trochanter to lift the femur for more extensive exposure. After insertion of implants and check of stability and length, the hip capsule was sutured, hemovac drain was not routinely placed and suture the wound in a standard layered fashion.

In group DAA, it utilizes a fracture table with the patient in the supine position. A skin incision, $8-12 \mathrm{~cm}$ long, was started about $3 \mathrm{~cm}$ lateral and distal to the anterior superior iliac spine and along the anterior margin of the tensor fasciae latae. The intermuscular space was opened bluntly between the sartorius muscles and tensor fasciae latae. The capsule was exposed anteriorly, a femoral neck osteotomy was performed. For femoral preparation, the posterior capsule was partial released and the greater trochanter could be elevated with a retractor to provide sufficient exposure. The femoral components was inserted in the same manner as that in group MIS-ALA with press fit fixation utilized. No redon drainage was intra-articularly placed, and routine closure was performed.

\section{Perioperative Interventions}

All participants received the same standardized preoperative and postoperative treatment, including pain management and rapid rehabilitation. All patients received prophylactic antibiotics and thromboprophylaxis. Physical therapy was initiated on the first postoperative day. All patients were allowed to abandon the crutches for full weight bearing to tolerance, depending on the individual immediate postoperative recovery and clinical condition. Patients were discharged from hospital after a minimum hospital stay of 2 days (range 2-6 days).

\section{Clinical Evaluations}

we recorded patients' background data and surgical data, including operating time, intraoperative blood loss, length of skin incision, etc. On postoperative day 1 hemoglobin $(\mathrm{Hb})$ was measured, and the number of blood transfusion was also recorded. Evaluations were conducted by two physical therapists blinded to the approaches employed at the following time points: preoperatively and postoperatively at 2,6 and 12 weeks. Clinical results were evaluated using the ability to climb stairs and walk, a 6 minutes walk test (6MWT) distances ${ }^{14}$, visual analog scale (VAS, $1-10$ point scale), the Forgotten Joint Scale (FJS-12), and Japanese Orthopaedic Association (JOA) Hip scores. The FJS-12 is a self-administered questionnaire that estimates patients' awareness of their knee or hip joint during activities of daily living, symbolizing a specific but very subjective patient-reported outcomes measure, and the eventual score range from 0 (worst) to 100 (best) ${ }^{15}$. JOA score has four subcategories: pain (Pain, 40 points), range of motion (ROM,20 points), ability to walk(Gait,20 points), and activities of daily living (ADL,20 points), a high JOA score is indicative of better hip function

${ }^{16}$. Presence of any other complications after operating were recorded during follow-up.

\section{Radiographic Evaluations}

Preoperative, 24 hours postoperative, 12 weeks postoperative, digitalized radiographs with an anteroposterior pelvis and cross-table lateral were performed routinely in all cases. Radiographs obtained at 12 weeks postoperative were examined to assess cup alignment (inclination angle, radiographic anteversion angle), and stem alignment (graded as valgus, neutral or varus) ${ }^{17}$ Anatomical anteversion evaluated in 24 hours postoperative computed tomography (CT) was converted into radiographic anteversion ${ }^{18}$. All radiographs were examined by two independent reviewers, they were not directly involved with patient treatment and thus were blinded from all clinical information during the assessment of radiographic implant alignment.

\section{Statistics}

We compared clinical outcomes and implant alignment between MIS-ALA and DAA groups. Continuous scales were compared with the two-sample t test or Wilcoxon's rank-sum test, categorical variables were compared by pearson's $\chi^{2}$ test or Fisher exact probability test using SPSS version 20.0 (SPSS Inc, Chicago, IL). With a p-value $<0.05$ considered statistically significant.

\section{Results}

\section{Clinical Outcomes}

Demographic data were comparable for the MIS-ALA and DAA groups (Table 1). There were no significant differences in age, gender, BMI, operative side, ASA status, Crowe classification, 6 MWT, preoperative VAS or preoperative JOA hip score between MIS-ALA and DAA groups (P >0.05). Surgical wounds, postoperative hospital stay, at all time points for postoperative VAS scores were also not significantly different $(P>0.05)$. (Table 2$)$ 
The surgery time was significantly shorter in the MIS-ALA group than in the group DAA [(62.4 \pm 9.05$) \min$ vs $(71 \pm 8.01)$ min, $\mathrm{P}<0.001]$. The blood loss for MIS-ALA subjects was $(132.6 \pm 43.31) \mathrm{ml}$ vs $(159.23 \pm 37.25) \mathrm{ml}$ for the DAA patients $(P=0.002)$. The Hb drop was also significantly lower on the first day postoperatively for MIS-ALA group $(P<0.001)$. 2(4.0\%) participants and $9(18.8 \%)$ participants in the MIS-ALA and DAA groups respectively required blood transfusions for anaemia, this difference was statistically significant $(P=0.021)$. (Table 2$)$

Postoperatively the MIS-ALA group had no significant differences who were using stairs normally, walking unlimited, $6 \mathrm{MWT}$ and wear shoes and socks with ease at 2, 6 and 12 weeks postoperatively compared to the DAA group ( $>0.05)$. In comparison of JOA scores in both groups 2 and 6 and 12 weeks after surgery, there were no statistically significant differences for any of the parameters measured $(P>0.05)$. In regards to patient-reported outcomes, the FJS-12 was significantly higher in the MIS-ALA group than in the group DAA at $2(P=0.006)$ and 6 weeks $(P=0.028)$ postoperatively $(64.5 \pm 17.96$ vs $55.52 \pm 13.18,74.9 \pm 13.98$ vs $68.65 \pm 13.84$, respectively). There was no significant difference in the FJS- 12 between the two groups at 12 weeks after surgery $(P=0.582)$. (Table 3$)$

\section{Radiological Outcomes}

There were no statistically significant differences in films positioning of implants between the MIS-ALA and DAA groups (P > 0.05; Table 2). On 12-week radiographs the MIS-ALA group had an average cup inclination of $41.96^{\circ} \pm 4.45$ compared to an average cup inclination for the DAA group was $40.71^{\circ}$ \pm 4.09 . Acetabular anteversion was $16.64^{\circ} \pm 5.63$ for the MIS-ALA and $15.92^{\circ} \pm 5.01$ for the DAA group respectively. No cups in either the MIS-ALA or DAA groups presented with a sign of migration at 12 weeks. The MIS-ALA group had 2 (4.0\%) stem implanted with varus compared to $3(6.2 \%)$ in the DAA group, but this difference was not statistically significant $(P=0.963)$. Femoral implants that subsided stabilized without radiological evidence of subsidence of $>3 \mathrm{~mm}$ in both groups at 12 weeks.

\section{Complications}

Surgical complications were described in Table 4. One fracture was identified with each group and managed conservatively. In the MIS-ALA group, a symptomatic partial fracture of the major trochanter postoperatively at identified 2 weeks due to a fall that did not require surgical fixation. In the DAA group, which was an intra-operative femoral perforation on broaching and was protected full weight-bearing for 6 weeks. The minor complication profile was inclined significantly to the DAA group as a result of the high incidence of lateral femoral cutaneous nerve (LFCN) neuropraxia. The leading symptom with the patient was dysesthesia of lateral thigh. The incidence of LFCN neuropraxia after surgery was 7(14.6\%) in the DAA group, zero occurred in the group MIS-ALA, this difference was statistically significant $(P=0.016)$. There were no complications of calcar fracture or postoperative dislocation discovered 12 weeks postoperatively. (Table 4)

\section{Discussion}

Primary early clinical outcomes following MIS-ALA and DAA THA were similar in this study. These measures including the ability to climb stairs and walk, 6MWT, VAS pain, and JOA scores. Hospital stay postoperative was also similar for both groups. The similar clinical outcomes suggest that pain relief and functional recovery over the total hip were not significantly different for both groups, which may be owe to the reduced invasiveness of both surgical approaches. Although the overall similarity of the MIS-ALA and DAA outcomes, some significant differences were confirmed in other domains. Early postoperative data, 2 and 6 weeks postoperative FJS-12 score were significantly better for the MIS-ALA group, this shows that better patientreported outcomes can be achieved by MIS-ALA THA. Nevertheless, the present study revealed that 12 weeks after surgery the FJS-12 score was similar between the MIS-ALA and DAA groups. Differences in favour of the MIS-ALA also included shorter operative times, less blood loss, lower Hb drop, fewer blood transfusions and LFCN neuropraxia.

The operation steps associated with the MIS-ALA, in part, explained the shorter surgical times with less blood loss, lower Hb drop, and fewer blood transfusions. The decreased blood loss was generally on the femoral side, related to an easier visualization of posterolateral capsular bleeders associated with the MIS-ALA. When THA was performed via the DAA, during elevation of the proximal femur release a portion of the posterolateral capsule and piriformis were at times necessary. Surgeon experience also played a great role, with over 100 MIS-ALA cases vs 200 DAA cases earlier to the study. However, the longer surgical time and more blood loss were not offset by better pain relief, faster function improved, and earlier discharge in the DAA group.

This study presented favorable implant alignments in THA with the both minimally invasive techniques. The MIS-ALA and DAA groups showed similar alignments both of cup and stem. Component positioning has a marked impact on the function and duration of THA ${ }^{19-21}$. The MIS-ALA and DAA surgical approaches can expose the hip joint intermuscular and internervous interval with the advantage of a lower chance of dislocation without higher revision risk ${ }^{22,23}$. Kawarai et $\mathrm{al}^{24}$ reported that cup anteversion in DAA nearly $4^{\circ}$ larger than MIS-ALA in the supine position. Inconsistent with our results. The larger anteversion in DAA could be due to the difference in rotation and tilt angles of the pelvis in the supine position. This could lead to the flexor muscles and femoral weight. During the cup procedure, the ipsilateral pelvis posteriorly could be retracted. According to Kobayashi et al ${ }^{25}$ research, it's a little difficult to insert the femoral stem in the position of neutral via DAA owing to the exposure of the proximal femur. Different with Kobayashi et al reports, the proximal femur could obtain adequate exposure to find the entry point in the DAA group and there was no statistically significant difference in stem implant alignment compared with the MIS-ALA group. The adequate exposure was obtained by longer surgical time to manage femur side.

The risk of minor surgical complications in our study favoured the MIS-ALA group due to the $14 \%$ incidence of LFCN neuropraxia in the DAA group. LFCN neuropraxia was a typical complication of DAA. Ozaki et al ${ }^{26,27}$ demonstrated that LFCN neuropraxia mainly resolves spontaneously over time after 
THA. The study's incidence was similar to previous reported range in the literature ${ }^{28-31}$. There were no connections between LFCN neuropraxia and $6 \mathrm{MWT}$ or JOA scores but had higher FJS-12 scores in MIS-ALA group. This was supported by Ozaki et al ${ }^{26}$.

This study has several limitations. First, it was an early evaluation of only 12 weeks after surgery. Longer follow-up was needed to compare the effects of the MIS-ALA and DAA methods on LFCN neuropraxia, FJS-12 scores, and implant alignment after THA. Second, the study could not be full blinded due to the visible difference in the surgical incisions. All radiographs review were blinded by two independent reviewers. In addition, only cementless stem was utilized. Generally, it was just needs narrower exposure of the proximal femur for a cementless stem to prepare the femur than did a cemented stem ${ }^{24,32}$. In this manner, minimally invasive surgery becomes less technically demanding and generates an advantage for the cementless stem.

\section{Conclusion}

Both MIS-ALA and DAA approaches yielded excellent and similar early clinical outcomes. However, some significant differences were confirmed in other domains. Better patient-reported outcomes could be achieved by MIS-ALA THA, as time went on, there were no significant differences in patient-reported outcomes between the two groups at 12 weeks after surgery. Differences in favour of the MIS-ALA included shorter operative times, less blood loss, lower $\mathrm{Hb}$ drop, fewer blood transfusions and LFCN neuropraxia.

\section{Abbreviations}

THA

Total Hip Arthroplasty

MIS-ALA

Minimally Anterolateral Approach

DAA

Direct Anterior Approach

6MWT

6 minutes walk test distances

FJS-12

Forgotten Joint Scale

JOA

Japanese Orthopaedic Association

LFCN

Lateral femoral cutaneous nerve

ASA

American Society of Anaesthesiologists grade

BMI

Body mass index

VAS

Visual analog scale

CT

Computed tomography

\section{Declarations}

\section{Acknowledgements}

The authors declare that the content of this article was composed in the absence of any financial or personal relationships that might inappropriately influence this study

\section{Authors' contributions}

$\mathrm{HL}$ and LY: wrote the paper. $\mathrm{HL}$ and JL: collected the data and participated in writing and revising the paper. SL and QT: performed all the analysis. JX: designed the whole study, completed all operations and helped to perform manuscript review. All authors read and approved the fnal manuscript.

\section{Funding}

This study was funded by Fujian Province Natural Science Foundation of China (2019J01173) and Special Research Project of Traditional Chinese Medicine of Sichuan Administration of China (No.2020JC0087)

\section{Code availability}

Not applicable. 


\section{Ethics approval and consent to participate}

This study was approved by Clinical Trials and Ethics Committee of Fujian Province Hospital. All patients provided consent for surgery and to have their data included in this study. All procedures performed in studies involving human participants were in accordance with the ethical standards of the institutional research committee and with the 1964 Helsinki Declaration and its later amendments or comparable ethical standards.

\section{Consent for publication}

All authors have stated for consents of publications. Availability of data and materials The datasets used and/or analyzed during the current study are fully available on reasonable request.

\section{Competing interests}

The authors declare that the content of this article was composed in the absence of any financial or personal relationships that might inappropriately influence this study

\section{References}

1. Anta-Díaz BD, Serralta-Gomis J, Lizaur-Utrilla A, Benavidez E, López-Prats FA. No differences between direct anterior and lateral approach for primary total hip arthroplasty related to muscle damage or functional outcome. International Orthopaedics 2016; 40 (10): 1-6.

2. Yang G, Chen W, Chen W, Tang X, Huang Y, Zhang L. Feasibility and Safety of 2-Day Discharge After Fast-Track Total Hip Arthroplasty: A Chinese Experience. Journal of Arthroplasty 2016; 31 (8): 1686-1692.e1681.

3. Kurtz SM, Lau E, Ong K, Zhao K, Kelly M, Bozic KJ. Future Young Patient Demand for Primary and Revision Joint Replacement: National Projections from 2010 to 2030. Clin Orthop Relat Res 2009; 24 (2): 2606-2612.

4. Dosanjh S, Matta JM, Bhandari M, Collaborative ftATR. The final straw: a qualitative study to explore patient decisions to undergo total hip arthroplasty. Archives of Orthopaedic \& Trauma Surgery 2009; 129 (6): 719-727.

5. Nakata K, Nishikawa M, Yamamoto K, Hirota S, Yoshikawa H. A Clinical Comparative Study of the Direct Anterior With Mini-Posterior Approach: Two Consecutive Series. Journal of Arthroplasty 2009; 24 (5): 698-704.

6. Rodriguez JA, Deshmukh AJ, Rathod PA, Greiz ML, Deshmane PP, Hepinstall MS, et al. Does the Direct Anterior Approach in THA Offer Faster Rehabilitation and Comparable Safety to the Posterior Approach? Clinical Orthopaedics \& Related Research® $2014 ; 472$ (2): 455-463.

7. Sugano N, Takao M, Sakai T, Nishii T, Miki H, Nakamura N. Comparison of mini-incision total hip arthroplasty through an anterior approach and a posterior approach using navigation. Orthop Clin North Am 2009; 40 (3): 365-370.

8. Smithpetersen MN. Approach to and exposure of the hip joint for mold arthroplasty. Journal of Bone \& Joint Surgery-american Volume 1949; 31 (1): 40-46.

9. Zlotnicki JP, O'Malley MJ. Direct Anterior Approach for Total Hip Arthroplasty: Implant, Instrument and Approach. Operative Techniques in Orthopaedics 2017; 27 (3): S1048666617300563.

10. Bertin KC, Rottinger H. Anterolateral mini-incision hip replacement surgery: a modified Watson-Jones approach. Clin Orthop Relat Res $2004 ; 429$ (429): 248-255.

11. Cheng TE, Wallis JA, Taylor NF, Holden CT, Marks P, Smith CL, et al. A Prospective Randomized Clinical Trial in Total Hip Arthroplasty-Comparing Early Results Between the Direct Anterior Approach and the Posterior Approach. Journal of Arthroplasty 2017; 32 (3): 883-890.

12. Jong LD, Klem TMAL, Kuijper TM, Roukema GR. The minimally invasive anterolateral approach versus the traditional anterolateral approach (Watson-Jones) for hip hemiarthroplasty after a femoral neck fracture: an analysis of clinical outcomes. International Orthopaedics $2018 ; 1-6$.

13. Repantis T,., Bouras T, ., Korovessis P,. Comparison of minimally invasive approach versus conventional anterolateral approach for total hip arthroplasty: a randomized controlled trial. European Journal of Orthopaedic Surgery \& Traumatology 2015; 25 (1): $111-116$.

14. Kennedy DM, Stratford PW, Susan R, Gollish JD. Using outcome measure results to facilitate clinical decisions the first year after total hip arthroplasty. Journal of Orthopaedic \& Sports Physical Therapy 2011; 41 (4): 232.

15. Behrend H, Giesinger K, Giesinger JM, Kuster MS. The "forgotten joint" as the ultimate goal in joint arthroplasty: validation of a new patient-reported outcome measure. Journal of Arthroplasty 2012; 27 (3): 430-436.e431.

16. Mibe J, Imakiire A, Watanabe T, Fujie T. Results of total hip arthroplasty with bone graft and support ring for protrusio acetabuli in rheumatoid arthritis. Journal of Orthopaedic Science 2005; 10 (1): 8-14.

17. Johnston RC, Fitzgerald RH, Harris WH, Poss R,., Müller ME, Sledge CB. Clinical and radiographic evaluation of total hip replacement. A standard system of terminology for reporting results. Journal of Bone \& Joint Surgery American Volume 1990; 72 (2): 161-168.

18. Nishii T, Sakai T, Takao M, Sugano N. Fluctuation of Cup Orientation During Press-Fit Insertion: A Possible Cause of Malpositioning. Journal of Arthroplasty 2015; 30 (10): 1847-1851.

19. Wan Z, Boutary M, Dorr LD. The Influence of Acetabular Component Position on Wear in Total Hip Arthroplasty. Journal of Arthroplasty 2008; 23 (1): 51-56. 
20. Sadhu A, Nam D, Coobs BR, Barrack T, Nunley RM, Barrack RL. Acetabular Component Position and the Risk of Dislocation Following Primary and Revision Total Hip Arthroplasty: A Matched Cohort Analysis. Journal of Arthroplasty 2017; 32 (3): 987-991.

21. Fujishiro T, Hayashi S, Kanzaki N, Hashimoto S, Shibanuma N, Kurosaka M. Effect of screw fixation on acetabular component alignment change in total hip arthroplasty. International Orthopaedics 2014; 38 (6): 1155-1158.

22. Sheth D, Cafri G, Inacio MCS, Paxton EW, Namba RS. Anterior and Anterolateral Approaches for THA Are Associated With Lower Dislocation Risk Without Higher Revision Risk. Clinical Orthopaedics \& Related Research® 2015; 473 (11): 3401-3408.

23. Mjaaland KE, Svenningsen S, Fenstad AM, Havelin LI, Furnes O, Nordsletten L. Implant Survival After Minimally Invasive Anterior or Anterolateral Vs. Conventional Posterior or Direct Lateral Approach: An Analysis of 21,860 Total Hip Arthroplasties from the Norwegian Arthroplasty Register (2008 to 2013). Journal of Bone \& Joint Surgery American Volume 2017; 99 (10): 840.

24. Kawarai Y, lida S, Nakamura J, Shinada Y, Suzuki C, Ohtori S. Does the surgical approach influence the implant alignment in total hip arthroplasty? Comparative study between the direct anterior and the anterolateral approaches in the supine position. International Orthopaedics 2017; 41 (12): 2487-2493.

25. Kobayashi H, Homma Y, Baba T, Ochi H, Matsumoto M, Yuasa T, et al. Surgeons changing the approach for total hip arthroplasty from posterior to direct anterior with fluoroscopy should consider potential excessive cup anteversion and flexion implantation of the stem in their early experience. International Orthopaedics 2016; 40 (9): 1-7.

26. Ozaki Y, Baba T, Homma Y, Ochi H, Watari T, Banno S, et al. Posterior versus direct anterior approach in total hip arthroplasty: difference in patientreported outcomes measured with the Forgotten Joint Score-12. SICOT-J.

27. Takada R, Jinno T, Miyatake K, Hirao M, Kimura A, Koga D, et al. Direct anterior versus anterolateral approach in one-stage supine total hip arthroplasty. Focused on nerve injury: A prospective, randomized, controlled trial. Journal of Orthopaedic Science Official Journal of the Japanese Orthopaedic Association 2018; S0949265818301404-.

28. Krista G, Beaulé PE, Kim PR, Anna F. Incidence of lateral femoral cutaneous nerve neuropraxia after anterior approach hip arthroplasty. Clinical Orthopaedics \& Related Research® 2010; 468 (9): 2397-2404.

29. Homma Y, Baba T, Sano K, Ochi H, Matsumoto M, Kobayashi H, et al. Lateral femoral cutaneous nerve injury with the direct anterior approach for total hip arthroplasty. International Orthopaedics 2016; 40 (8): 1587-1593.

30. Ozaki Y, Homma Y, Sano K, Baba T, Ochi H, Desroches A, et al. Small femoral offset is a risk factor for lateral femoral cutaneous nerve injury during total hip arthroplasty using a direct anterior approach. Orthopaedics \& Traumatology Surgery \& Research $2016 ; 102$ (8): 740.

31. Patton RS, Runner RP, Lyons RJ, Bradbury TL. Clinical Outcomes of Patients With Lateral Femoral Cutaneous Nerve Injury After Direct Anterior Total Hip Arthroplasty. Journal of Arthroplasty 2018.

32. Yuki M, Nobuhiko S, Nobuo N, Makoto H. The Accuracy of a Mechanical Cup Alignment Guide in Total Hip Arthroplasty (THA) Through Direct Anterior and Posterior Approaches Measured with CT-Based Navigation. Journal of Arthroplasty 2015; 30 (9): 1561-1564.

\section{Tables}

Table 1 Demographic Data on the Patients.

\begin{tabular}{|llll|}
\hline Variable & MIS-ALA & DAA & $P$-Value \\
\hline Age $(\mathrm{y})$ & $62.44 \pm 7.07$ & $62.21 \pm 8.22$ & 0.881 \\
\hline Males/Females $(\mathrm{n})$ & $17(34.0 \%) / 33(66.0 \%)$ & $23(47.9 \%) / 25(52.1 \%)$ & 0.161 \\
\hline BMI $\left(\mathrm{kg} / \mathrm{m}^{2}\right)$ & $22.46 \pm 4.23$ & $22.96 \pm 3.11$ & 0.509 \\
\hline Operative side - right $(\mathrm{n})$ & $23(46.0 \%)$ & $25(52.1 \%)$ & 0.547 \\
\hline ASA score (l:II:III) & $11 / 30 / 9$ & $9 / 34 / 5$ & 0.460 \\
\hline Crowe (I:II:III:IV) & $13: 34: 3$ & $14: 31: 3$ & 0.935 \\
\hline 6 MWT (m) & $289 \pm 51.36$ & $279.17 \pm 57.05$ & 0.372 \\
\hline Pre-op VAS (points) & $7.32 \pm 1.02$ & $7.23 \pm 1.12$ & 0.675 \\
\hline Pre-op JOA (points) & & & 0.991 \\
\hline Pain & $16.74 \pm 4.58$ & $16.73 \pm 5.37$ & 0.227 \\
\hline ROM & $12.98 \pm 2.99$ & $12.21 \pm 3.29$ & 0.494 \\
\hline Gait & $10.54 \pm 3.45$ & $11.02 \pm 3.49$ & 0.160 \\
\hline ADL & $11.32 \pm 3.63$ & $10.35 \pm 3.08$ & 0.521 \\
\hline Total & $51.58 \pm 9.69$ & $50.31 \pm 9.77$ & \\
\hline
\end{tabular}


Table 2 Surgical and Immediate Post-Operative Data

\begin{tabular}{|llll|}
\hline Variable & MIS-ALA & DAA & $P$-Value \\
\hline Surgery time (min) & $62.4 \pm 9.05$ & $71 \pm 8.01$ & $<0.001$ \\
\hline Incision (cm) & $9.86 \pm 0.99$ & $10.04 \pm 1.07$ & 0.385 \\
\hline Blood loss (ml) & $132.6 \pm 43.31$ & $159.23 \pm 37.25$ & 0.002 \\
\hline Hb drop (g/L) & $29.56 \pm 8.02$ & $36.4 \pm 7.12$ & $<0.001$ \\
\hline Blood transfusion (n) & $2(4.0 \%)$ & $9(18.8 \%)$ & 0.021 \\
\hline post-op hospital stay (day) & $4.6 \pm 1.14$ & $4.77 \pm 1.17$ & 0.467 \\
\hline VAS (points) & & & \\
\hline 1 day post-op & $4.2 \pm 0.99$ & $4.38 \pm 0.82$ & 0.343 \\
\hline 3 day post-op & $2.82 \pm 0.83$ & $2.79 \pm 0.8$ & 0.863 \\
\hline 7 day post-op & $2.24 \pm 0.52$ & $2.38 \pm 0.57$ & 0.222 \\
\hline Cup Inclination $\left(^{\circ}\right)$ & $41.96 \pm 4.45$ & $40.71 \pm 4.09$ & 0.151 \\
\hline Cup Anteversion $\left(^{\circ}\right)$ & $16.64 \pm 5.63$ & $15.92 \pm 5.01$ & 0.504 \\
\hline Femoral Stem Position (n) & & & 0.963 \\
\hline Valgus & $0(0 \%)$ & $0(0 \%)$ & \\
\hline Neutral & $48(96.0 \%)$ & $45(93.8 \%)$ & \\
\hline Varus & $2(4.0 \%)$ & $3(6.2 \%)$ & \\
\hline
\end{tabular}

Table 3 Post-Operative Outcomes data

\begin{tabular}{|c|c|c|c|c|c|c|c|c|c|}
\hline \multirow[t]{2}{*}{ Variable } & \multicolumn{3}{|l|}{2 Weeks } & \multicolumn{3}{|l|}{6 Weeks } & \multicolumn{3}{|l|}{12 Weeks } \\
\hline & MIS-ALA & DAA & $\begin{array}{l}P \text { - } \\
\text { Value }\end{array}$ & MIS-ALA & DAA & $\begin{array}{l}P \text { - } \\
\text { Value }\end{array}$ & MIS-ALA & DAA & $P$-Value \\
\hline VAS (points) & $2.16 \pm 1.2$ & $2.29 \pm 1.32$ & 0.607 & $1.86 \pm 1.16$ & $1.85 \pm 1.24$ & 0.981 & $1.4 \pm 1.14$ & $1.13 \pm 0.84$ & 0.180 \\
\hline Stairs Normally (n) & $16(32.0 \%)$ & $14(29.2 \%)$ & 0.761 & $24(48.0 \%)$ & $26(54.2 \%)$ & 0.542 & $42(84.0 \%)$ & $36(75.0 \%)$ & 0.269 \\
\hline $\begin{array}{l}\text { Distance Unlimited } \\
\text { (n) }\end{array}$ & $25(50.0 \%)$ & $20(41.7 \%)$ & 0.408 & $38(76.0 \%)$ & $39(81.3 \%)$ & 0.527 & $42(84.0 \%)$ & $42(87.5 \%)$ & 0.621 \\
\hline $\begin{array}{l}\text { Shoes and Socks } \\
\text { with Ease (n) }\end{array}$ & $21(42.0 \%)$ & $24(50.0 \%)$ & 0.427 & $36(72.0 \%)$ & $32(66.7 \%)$ & 0.567 & $42(84.0 \%)$ & $43(89.6 \%)$ & 0.415 \\
\hline 6MWT(m) & $225 \pm 87.74$ & $238.54 \pm 86.17$ & 0.443 & $346.6 \pm 86.26$ & $332.29 \pm 72.24$ & 0.377 & $410.6 \pm 82.69$ & $425.83 \pm 86.44$ & 0.375 \\
\hline FJS-12 & $64.5 \pm 17.96$ & $55.52 \pm 13.18$ & 0.006 & $74.9 \pm 13.98$ & $68.65 \pm 13.84$ & 0.028 & $80.2 \pm 13.21$ & $78.54 \pm 16.44$ & 0.582 \\
\hline \multicolumn{10}{|l|}{ JOA (points) } \\
\hline Pain & $29.2 \pm 4.5$ & $30.81 \pm 5.17$ & 0.102 & $36.76 \pm 2.54$ & $36.02 \pm 2.42$ & 0.143 & $38.98 \pm 1.8$ & $38.44 \pm 2.61$ & 0.232 \\
\hline ROM & $13.88 \pm 3.27$ & $14.5 \pm 3.59$ & 0.373 & $16.28 \pm 1.33$ & $16.52 \pm 2.41$ & 0.539 & $16.96 \pm 1.73$ & $17.38 \pm 1.95$ & 0.267 \\
\hline Gait & $13.6 \pm 2.96$ & $12.94 \pm 3.48$ & 0.312 & $14.1 \pm 3.87$ & $15.33 \pm 3.22$ & 0.090 & $18.06 \pm 1.75$ & $17.42 \pm 2.56$ & 0.148 \\
\hline$A D L$ & $13.9 \pm 3.02$ & $14.44 \pm 2.7$ & 0.356 & $15.04 \pm 3.52$ & $15.88 \pm 3.55$ & 0.245 & $16.76 \pm 1.51$ & $17.25 \pm 1.64$ & 0.127 \\
\hline Total & $70.58 \pm 7.49$ & $72.69 \pm 9.09$ & 0.213 & $82.52 \pm 7.62$ & $84.33 \pm 6.77$ & 0.217 & $90.76 \pm 3.16$ & $90.48 \pm 6.97$ & 0.797 \\
\hline
\end{tabular}

Table 4 Surgical complications 


\begin{tabular}{|llll|}
\hline Complication & MIS-ALA & DAA & $P$-Value \\
\hline Superficial infection (hips) & 0 & 0 & 1.00 \\
\hline Deep infection & 0 & 0 & 1.00 \\
\hline DVT & 0 & 0 & 1.00 \\
\hline Calcar fracture & 0 & 0 & 1.00 \\
\hline Major trochanteric fracture & $1(2 \%)$ & $1(2.1 \%)$ & 1.00 \\
\hline LCFN neuropraxia & 0 & $7(14.6 \%)$ & 0.016 \\
\hline Nerve palsy & 0 & 0 & 1.00 \\
\hline Post-operative dislocation & 0 & 0 & 1.00 \\
\hline Post-operative femoral fracture & 0 & 0 & 1.00 \\
\hline Cup migration & 0 & 0 & 1.00 \\
\hline Stem subsidence $>3$ mm & 0 & 0 & 1.00 \\
\hline Thigh pain & 0 & 0 & 1.00 \\
\hline Bursitis & 0 & 0 & 1.00 \\
\hline
\end{tabular}

